\title{
The Reissner-Nordström black hole with the fastest relaxation rate
}

\author{
Shahar Hod ${ }^{1,2, a}$ \\ ${ }^{1}$ The Ruppin Academic Center, 40250 Emeq Hefer, Israel \\ 2 The Hadassah Academic College, 91010 Jerusalem, Israel
}

Received: 12 September 2018 / Accepted: 5 November 2018 / Published online: 14 November 2018

(C) The Author(s) 2018

\begin{abstract}
Numerous numerical investigations of the quasinormal resonant spectra of Kerr-Newman black holes have revealed the interesting fact that the characteristic relaxation times $\tau(\bar{a}, \bar{Q})$ of these canonical black-hole spacetimes can be described by a two-dimensional function $\bar{\tau} \equiv \tau / M$ which increases monotonically with increasing values of the dimensionless angular-momentum parameter $\bar{a} \equiv J / M^{2}$ and, in addition, is characterized by a non-trivial (non-monotonic) functional dependence on the dimensionless charge parameter $\bar{Q} \equiv Q / M$. In particular, previous numerical investigations have indicated that, within the family of spherically symmetric charged ReissnerNordström spacetimes, the black hole with $\bar{Q} \simeq 0.7$ has the fastest relaxation rate. In the present paper we use analytical techniques in order to investigate this intriguing non-monotonic functional dependence of the ReissnerNordström black-hole relaxation rates on the dimensionless physical parameter $\bar{Q}$. In particular, it is proved that, in the eikonal (geometric-optics) regime, the black hole with $\bar{Q}=\frac{\sqrt{51-3 \sqrt{33}}}{8} \simeq 0.73$ is characterized by the fastest relaxation rate (the smallest dimensionless relaxation time $\bar{\tau}$ ) within the family of charged Reissner-Nordström blackhole spacetimes.
\end{abstract}

\section{Introduction}

The mathematically elegant uniqueness theorems of Israel, Carter, and Hawking [1-4] (see also [5-7]) have revealed the physically important fact that all asymptotically flat stationary black-hole solutions of the non-linearly coupled EinsteinMaxwell field equations belong to the Kerr-Newman family of curved spacetimes [8-41]. The three-dimensional phasespace of these canonical black-hole spacetimes is described by conserved physical parameters that can be measured by

a e-mail: shaharhod@gmail.com asymptotic observers: the black-hole mass $M$, the black-hole electric charge $Q$, and the black-hole angular momentum $J \equiv M a$.

In accord with the results of the uniqueness theorems [1,37], the linearized dynamics of massless gravitational and electromagnetic fields in perturbed Kerr-Newman black-hole spacetimes are characterized by damped quasinormal resonant modes with the physically motivated boundary conditions of purely ingoing waves at the black-hole outer horizon and purely outgoing waves at spatial infinity [42]. These exponentially decaying black-hole-field oscillation modes describe the gradual dissipation of the external massless perturbation fields which are radiated into the black hole and to spatial infinity. As a result of this dissipation process, the perturbed spacetime gradually returns into a stationary KerrNewman black-hole solution of the Einstein-Maxwell field equations.

Interestingly, for a given set $\left\{\bar{Q} \equiv Q / M, \bar{a} \equiv J / M^{2}\right\}$ of the Kerr-Newman dimensionless physical parameters $[43,44]$, the physically motivated boundary conditions at the black-hole horizon and at spatial infinity single out a discrete set $\{\bar{\omega} \equiv M \omega(\bar{Q}, \bar{a} ; l, m, n)\}_{n=0}^{n=\infty}[45]$ of dimensionless complex resonant frequencies which characterize the relaxation dynamics of the massless fields in the curved black-hole spacetime (see [46-48] for excellent reviews on the physically exciting topic of black-hole resonant spectra). In particular, the fundamental resonant frequency $\bar{\omega}(n=0)$ [49] determines, through the relation

$\bar{\tau}_{\text {relax }} \equiv \bar{\omega}_{\mathrm{I}}^{-1}(n=0)$,

the characteristic dimensionless relaxation time of the perturbed black-hole spacetime.

One can ask: Within the family of charged and rotating Kerr-Newman black-hole spacetimes, which black hole has the longest relaxation time $\bar{\tau}_{\text {relax }}(\bar{Q}, \bar{a})$ ? Interestingly, it has previously been proved that the answer to this question is 
not unique. In particular, previous analytical [50-55] and numerical [56-61] studies of the Kerr and Kerr-Newman quasinormal resonance spectra have explicitly proved that the characteristic black-hole relaxation times grow unboundedly as the extremal limit $\bar{T}_{\mathrm{BH}} \rightarrow 0$ is approached [62]:

$\bar{\tau}_{\text {relax }} \propto \bar{T}_{\mathrm{BH}}^{-1} \rightarrow \infty \quad$ for $\quad \bar{T}_{\mathrm{BH}} \rightarrow 0$

where $\bar{T}_{\mathrm{BH}} \equiv M T_{\mathrm{BH}}$ is the characteristic dimensionless Bekenstein-Hawking temperature of the black-hole spacetime [63-65].

In the present paper we shall focus on the opposite regime of Reissner-Nordström black holes which minimize the dimensionless relaxation time (1). In particular, we here raise the following physically interesting question: Within the family of spherically symmetric charged Reissner-Nordström black holes, which black hole has the fastest relaxation rate [that is, the shortest relaxation time $\bar{\tau}_{\text {relax }}(\bar{Q})$ ]? Intriguingly, below we shall explicitly prove that, as opposed to the case of near-extremal spacetimes which maximize the black-hole relaxation times [see Eq. (2)], the answer to the black-hole minimization question is unique.

\section{Review of former analytical and numerical studies}

Former analytical and numerical studies of the Kerr-Newman resonance spectra [50-61] have revealed the fact that, for a given value of the black-hole electric charge $\bar{Q}$, the characteristic black-hole relaxation time $\bar{\tau}_{\text {relax }}(\bar{Q}, \bar{a})$ is a monotonically increasing function of the rotation parameter $\bar{a}$. Hence, within the canonical family of Kerr-Newman blackhole spacetimes, the relaxation rate $\bar{\tau}_{\text {relax }}^{-1}(\bar{Q}, \bar{a})$ can be maximized by considering non-spinning $(\bar{a}=0)$ black holes [66]. In the present paper we shall therefore focus our attention on spherically symmetric charged Reissner-Nordström blackhole spacetimes.

In addition, former numerical studies [56,60,61,67-69] of the resonance spectra of charged black holes have revealed the interesting fact that the black-hole relaxation times $\bar{\tau}_{\text {relax }}(\bar{Q}, \bar{a})$ are characterized by a non-trivial (nonmonotonic) functional dependence on the dimensionless physical parameter $\bar{Q}$. In particular, detailed numerical computations $[57,58,69]$ indicate that, in the physically interesting case of coupled gravitational-electromagnetic quadrupole perturbations fields with $l=2$, the black-hole relaxation time (1) is minimized for

$\bar{Q}_{\min } \simeq 0.7$ for $l=2$.

The main goal of the present compact paper is to shed light on the intriguing non-monotonic functional dependence of the characteristic Reissner-Nordström black-hole relaxation times $\bar{\tau}_{\text {relax }}(\bar{Q})$ on the dimensionless charge parameter $\bar{Q}$. In particular, in order to facilitate a fully analytical treatment of the black-hole minimization problem, we shall study in the present paper the eikonal (geometric-optics) regime of the quasinormal resonance spectra which characterize the composed black-hole-field system. Interestingly, below we shall explicitly demonstrate that the analytical results obtained in the eikonal $l \gg 1$ regime agree remarkably well with the numerical results $[57,58,69]$ of the quadrupole $l=2$ perturbation fields.

\section{The composed black-hole-field quasinormal resonance spectra in the eikonal (geometric-optics) $l \gg 1$ regime}

We consider a Reissner-Nordström black-hole spacetime of mass $M$ and electric charge $Q$ which is characterized by the curved line element $[8,43]$

$d s^{2}=-f(r) d t^{2}+\frac{1}{f(r)} d r^{2}+r^{2}\left(d \theta^{2}+\sin ^{2} \theta d \phi^{2}\right)$

where

$f(r)=1-\frac{2 M}{r}+\frac{Q^{2}}{r^{2}}$.

The radii [8]

$r_{ \pm}=M \pm \sqrt{M^{2}-Q^{2}}$

of the inner and outer horizons of the charged black-hole spacetime (4) are determined by the zeroes of the radiallydependent metric function (5)

Massless perturbations fields of the black-hole spacetime (4) are governed by the Schrödinger-like ordinary differential equation [8]

$\frac{d^{2} \psi}{d y^{2}}+V_{l}[r(y)] \psi=0$

where the tortoise radial coordinate $y$ is defined by the differential relation

$d y=\frac{d r}{f(r)}$

and, in the eikonal (geometric-optics) $l \gg 1$ limit, the effective black-hole-field curvature potential in (7) is given by the functional relation [8]

$V_{l}(r)=\omega^{2}-l(l+1) \frac{f(r)}{r^{2}}$. 
The quasinormal resonant modes (damped oscillations) which characterize the linearized relaxation dynamics of the perturbed black-hole spacetime are determined by the Schrödinger-like radial equation (7) with the physically motivated boundary conditions of purely ingoing waves at the black-hole event horizon [which is characterized by $y \rightarrow$ $-\infty$ ] and purely outgoing waves at spatial infinity [which is characterized by $y \rightarrow \infty]$ :

$\psi \sim \begin{cases}e^{-i \omega y} & \text { for } r \rightarrow r_{+}(y \rightarrow-\infty) \\ e^{i \omega y} & \text { for } r \rightarrow \infty \quad(y \rightarrow \infty) .\end{cases}$

We shall now use analytical techniques in order to determine the characteristic quasinormal resonant frequencies which characterize the black-hole spacetime (4) in the eikonal large$l$ regime. To this end, we shall use the results of the elegant WKB analysis presented in [70,71], according to which the quasinormal resonance spectrum which characterizes the Schrödinger-like ordinary differential equation (7) with the boundary conditions (10) is determined, in the eikonal $l \gg 1$ regime, by the simple leading-order resonance equation $[70,71]$

$i \frac{V_{0}}{\sqrt{2 V_{0}^{(2)}}}=n+\frac{1}{2}+O\left(l^{-1}\right) ; \quad n=0,1,2, \ldots$

Here the composed black-hole-field curvature potential $V_{0}$ and its radial derivatives $V_{0}^{(k)} \equiv d^{k} V / d y^{k}$ [see Eq. (9)] are evaluated at the radial peak $y=y_{0}$, which is characterized by the simple functional relation

$\frac{d V}{d y}=0$ for $y=y_{0}\left(r=r_{0}\right)$

Substituting the effective black-hole-field radial potential (9) into (12), one finds the simple expression [69]

$\bar{r}_{0} \equiv \frac{r_{0}}{M}=\frac{1}{2}\left(3+\sqrt{9-8 \bar{Q}^{2}}\right)$

for the dimensionless radius which characterizes the peak of the curvature potential in the eikonal large- $l$ regime. Substituting (13) into the WKB resonance equation (11) and using the differential relation (8), one obtains the (rather cumbersome) functional expressions

$\bar{\omega}_{\mathrm{R}}=\frac{(2 l+1) \sqrt{2} \sqrt{3+\sqrt{9-8 \bar{Q}^{2}}-2 \bar{Q}^{2}}}{\left[3+\sqrt{9-8 \bar{Q}^{2}}\right]^{2}}$ and

$$
\begin{aligned}
\bar{\omega}_{\mathrm{I}}= & -\left(n+\frac{1}{2}\right) \\
& \cdot \frac{4 \sqrt{3} \sqrt{\left[3+\sqrt{9-8 \bar{Q}^{2}}-2 \bar{Q}^{2}\right]\left[3+\sqrt{9-8 \bar{Q}^{2}}-\frac{8}{3} \bar{Q}^{2}\right]}}{\left[3+\sqrt{9-8 \bar{Q}^{2}}\right]^{3}} ; \\
& n=0,1,2, \ldots
\end{aligned}
$$

for the characteristic dimensionless resonant frequencies of the charged Reissner-Nordström black-hole spacetime (4).

Inspecting the analytically derived geometric-optics expression (15), one reveals the interesting fact that the function $\bar{\omega}_{\mathrm{I}}(\bar{Q})$ has a non-trivial (non-monotonic) dependence on the dimensionless physical parameter $\bar{Q}$. In particular, analyzing the function $\bar{\omega}_{\mathrm{I}}^{(0)}(\bar{Q})$ [see Eq. (15)] for the fundamental $(n=0)$ resonant frequency in the eikonal regime, we find that the characteristic dimensionless relaxation time $\bar{\tau}(\bar{Q}) \equiv 1 / \bar{\omega}_{\mathrm{I}}^{(0)}$ of the composed black-hole-field system is minimized for the particular value

$\bar{Q}_{\text {min }}=\frac{\sqrt{51-3 \sqrt{33}}}{8} \simeq 0.726$ for $l \gg 1$

of the dimensionless black-hole charge parameter.

Interestingly, one finds that the analytically derived expression (16) for the dimensionless charge parameter $\bar{Q}_{\text {min }}$, which characterizes the Reissner-Nordström black hole with the fastest relaxation rate (the shortest relaxation time) in the eikonal large- $l$ regime, agrees remarkably well with the numerically computed value $\bar{Q}_{\text {min }}^{\text {numerical }} \simeq 0.7[56-$ 61,69] [see Eq. (3)] for the quadrupole $l=2$ perturbation fields.

\section{Summary and discussion}

The characteristic quasinormal resonance spectra of black holes have attracted the attention of physicists and mathematicians during the last five decades [46-48]. In the present paper we have raised the following physically interesting questions: Within the canonical family of charged and rotating Kerr-Newman black holes, which black hole has the slowest relaxation rate and which black hole has the fastest relaxation rate?

Using analytical techniques we have shown that, while the answer to the first question is not unique [see the characteristic relation (2) for the family of near-extremal black holes with diverging relaxation times in the $\bar{T}_{\mathrm{BH}} \rightarrow 0$ limit], the answer to the second question is unique within the phase space of spherically symmetric charged ReissnerNordström black-hole spacetimes. In particular, based on previous analytical and numerical studies of the black-hole 
resonance spectra [50-61] which revealed the interesting fact that, for a given value of the dimensionless charge parameter $\bar{Q}$, the characteristic black-hole relaxation time $\bar{\tau}(\bar{a}, \bar{Q})$ is a monotonically increasing function of the dimensionless angular-momentum parameter $\bar{a}$, we have focused our attention on the relaxation properties of non-spinning $(\bar{a}=0)$ charged Reissner-Nordström black-hole spacetimes. In addition, in order to facilitate a fully analytical exploration of the intriguing non-monotonic functional dependence of the black-hole relaxation rates $\bar{\tau}^{-1}(\bar{Q})$ on the dimensionless physical parameter $\bar{Q}$, we have considered in the present paper the eikonal (geometric-optics) regime of the composed black-hole-field resonance spectrum.

Using standard analytical techniques [70,71], we have explicitly demonstrated that the composed black-hole-field relaxation spectrum is characterized by a non-trivial (nonmonotonic) functional dependence on the dimensionless charge parameter $\bar{Q}$. This analytically derived result supports the numerical data presented in [57,58,69]. Moreover, it is interesting to note that the analytically calculated black-hole charge parameter [see Eq. (16)]

$$
\left(\frac{Q}{M}\right)_{\min }^{\text {analytical }}=\frac{\sqrt{51-3 \sqrt{33}}}{8},
$$

which minimizes the dimensionless relaxation time of the composed Reissner-Nordström-black-hole-field system in the eikonal $l \gg 1$ regime, agrees remarkably well with the numerically computed charge parameter $\bar{Q}_{\text {min }}^{\text {numerical }} \simeq$ 0.7 [see Eq. (3)] which minimizes the black-hole relaxation time in the canonical case of coupled gravitationalelectromagnetic quadrupole perturbation fields with $l=2$ $[57,58,69]$.

Acknowledgements This research is supported by the Carmel Science Foundation. I would like to thank Yael Oren, Arbel M. Ongo, Ayelet B. Lata, and Alona B. Tea for helpful discussions.

Open Access This article is distributed under the terms of the Creative Commons Attribution 4.0 International License (http://creativecomm ons.org/licenses/by/4.0/), which permits unrestricted use, distribution, and reproduction in any medium, provided you give appropriate credit to the original author(s) and the source, provide a link to the Creative Commons license, and indicate if changes were made. Funded by SCOAP ${ }^{3}$.

\section{References}

1. W. Israel, Phys. Rev. 164, 1776 (1967)

2. W. Israel, Commun. Math. Phys. 8, 245 (1968)

3. B. Carter, Phys. Rev. Lett. 26, 331 (1971)

4. S.W. Hawking, Commun. Math. Phys. 25, 152 (1972)

5. D.C. Robinson, Phys. Rev. D 10, 458 (1974)

6. D.C. Robinson, Phys. Rev. Lett. 34, 905 (1975)

7. J. Isper, Phys. Rev. Lett. 27, 529 (1971)
8. S. Chandrasekhar, The mathematical theory of black holes (Oxford University Press, New York, 1983)

9. R.P. Kerr, Phys. Rev. Lett. 11, 237 (1963)

10. E.T. Newman, R. Couch, K. Chinnapared, A. Exton, A. Prakash et al., J. Math. Phys. 6, 918 (1965)

11. See [12-41] for the physically interesting case of non KerrNewman black-hole solutions of the coupled Einstein-Maxwellscalar field equations which describe spinning black holes that support linear [12-22] and non-linear [23-41] stationary scalar (bosonic) fields.

12. S. Hod, Phys. Rev. D 86, 104026 (2012). arXiv: 1211.3202

13. S. Hod, Euro. Phys. J. C 73, 2378 (2013). arXiv:1311.5298

14. S. Hod, Phys. Rev. D 90, 024051 (2014). arXiv:1406.1179

15. S. Hod, Phys. Lett. B 739, 196 (2014). arXiv:1411.2609

16. S. Hod, Class. Quant. Grav. 32, 134002 (2015). arXiv: 1607.00003

17. S. Hod, Phys. Lett. B 751, 177 (2015)

18. S. Hod, Class. Quant. Grav. 33, 114001 (2016)

19. S. Hod, Phys. Lett. B 758, 181 (2016). arXiv:1606.02306

20. S. Hod, O. Hod, Phys. Rev. D 81, 061502 Rapid communication (2010). arXiv:0910.0734

21. S. Hod, Phys. Lett. B 708, 320 (2012). arXiv:1205.1872

22. S. Hod, J. High Energy Phys. 01, 030 (2017). arXiv:1612.00014

23. C.A.R. Herdeiro, E. Radu, Phys. Rev. Lett. 112, 221101 (2014)

24. C.L. Benone, L.C.B. Crispino, C. Herdeiro, E. Radu, Phys. Rev. D 90, 104024 (2014)

25. C.A.R. Herdeiro, E. Radu, Phys. Rev. D 89, 124018 (2014)

26. C.A.R. Herdeiro, E. Radu, Int. J. Mod. Phys. D 23, 1442014 (2014)

27. Y. Brihaye, C. Herdeiro, E. Radu, Phys. Lett. B 739, 1 (2014)

28. J.C. Degollado, C.A.R. Herdeiro, Phys. Rev. D 90, 065019 (2014)

29. C. Herdeiro, E. Radu, H. Rúnarsson, Phys. Lett. B 739, 302 (2014)

30. C. Herdeiro, E. Radu, Class. Quantum Grav. 32, 144001 (2015)

31. C.A.R. Herdeiro, E. Radu, Int. J. Mod. Phys. D 24, 1542014 (2015)

32. C.A.R. Herdeiro, E. Radu, Int. J. Mod. Phys. D 24, 1544022 (2015)

33. P.V.P. Cunha, C.A.R. Herdeiro, E. Radu, H.F. Rúnarsson, Phys. Rev. Lett. 115, 211102 (2015)

34. B. Kleihaus, J. Kunz, S. Yazadjiev, Phys. Lett. B 744, 406 (2015)

35. C.A.R. Herdeiro, E. Radu, H.F. Rúnarsson, Phys. Rev. D 92 , 084059 (2015)

36. C. Herdeiro, J. Kunz, E. Radu, B. Subagyo, Phys. Lett. B 748, 30 (2015)

37. C.A.R. Herdeiro, E. Radu, H.F. Rúnarsson, Class. Quant. Grav. 33, 154001 (2016)

38. C.A.R. Herdeiro, E. Radu, H.F. Rúnarsson, Int. J. Mod. Phys. D 25, 1641014 (2016)

39. Y. Brihaye, C. Herdeiro, E. Radu, Phys. Lett. B 760, 279 (2016)

40. Y. Ni, M. Zhou, A.C. Avendano, C. Bambi, C.A.R. Herdeiro, E. Radu, JCAP 1607, 049 (2016)

41. M. Wang. arXiv: 1606.00811

42. S.L. Detweiler, in Sources of Gravitational Radiation, ed. by L. Smarr (Cambridge University Press, Cambridge, 1979)

43. We shall use natural units in which $G=c=\hbar=1$.

44. We shall assume without loss of generality that $\bar{Q} \geq 0$.

45. The dimensionless parameters $\{l, m\}$ are respectively the spheroidal harmonic index and the azimuthal harmonic index of the massless perturbation modes.

46. H.P. Nollert, Class. Quant. Grav. 16, R159 (1999)

47. E. Berti, V. Cardoso, A.O. Starinets, Class. Quant. Grav. 26, 163001 (2009)

48. R.A. Konoplya, A. Zhidenko, Rev. Mod. Phys. 83, 793 (2011)

49. The fundamental resonant mode of the black-hole spacetime is defined as the mode with the smallest imaginary part (the perturbation mode with the largest relaxation time).

50. S. Hod, Phys. Rev. D 75, 064013 (2007). arXiv:gr-qc/0611004

51. S. Hod, Class. Quant. Grav. 24, 4235 (2007). arXiv:0705.2306

52. A. Gruzinov. arXiv:gr-qc/0705.1725

53. S. Hod, Phys. Rev. D 78, 084035 (2008). arXiv:0811.3806 
54. S. Hod, Phys. Lett. B 666, 483 (2008). arXiv:0810.5419

55. S. Hod, Phys. Lett. B 715, 348 (2012). arXiv:1207.5282

56. E. Berti, K.D. Kokkotas, Phys. Rev. D 71, 124008 (2005)

57. P. Pani, E. Berti, L. Gualtieri, Phys. Rev. Lett. 110, 241103 (2013)

58. P. Pani, E. Berti, L. Gualtieri, Phys. Rev. D 88, 064048 (2013)

59. S. Hod, Euro. Phys. J. C 75, 272 (2015). arXiv:1410.2252

60. O.J.C. Dias, M. Godazgar, J.E. Santos, Phys. Rev. Lett. 114, 151101 (2015)

61. S. Hod, Euro. Phys. J. C 75, 611 (2015). arXiv: 1511.05696

62. It is worth emphasizing that the simple near-extremal functional behavior (2) has been proved analytically for generic massless perturbation fields of near-extremal neutral spinning Kerr black holes [50-55]. In addition, this functional relation has been proved analytically for the case of scalar perturbation fields of near-extremal charged spinning Kerr-Newman black-hole spacetimes [50-55]. For the case of coupled gravitational-electromagnetic perturbations of the Kerr-Newman black-hole spacetime, the functional relation (2) has been demonstrated numerically in the dimensionless charge regime $Q / r_{+} \leq 0.9$ [here $r_{+}$is the horizon radius of the black-hole spacetime, see Eq. (6) below] [56-61].

63. The Bekenstein-Hawking temperature of the Kerr-Newman black hole is given by the functional expression $T_{\mathrm{BH}}=\left(r_{+}-\right.$ $\left.r_{-}\right) / 4 \pi\left(r_{+}^{2}+a^{2}\right)$, where $r_{ \pm}$are the horizon radii of the black-hole spacetime [see Eq. (6)below].
64. J.D. Bekenstein, Phys. Rev. D 7, 2333 (1973)

65. S.W. Hawking, Commun. Math. Phys. 43, 199 (1975)

66. It is worth noting that, for Kerr-Newman black holes, the qualitative difference in the functional behavior of the relaxation time between the case in which the charge is varied and the case in which the spin parameter is varied is probably related to the fact that the null circular geodesic of a near-extremal Kerr black hole with $a / M \rightarrow$ $1^{-}$is located in the vicinity of the black-hole horizon, whereas the null circular geodesic of a near-extremal Reissner-Nordström black hole with $Q / M \rightarrow 1^{-}$is located a finite radial distance from the black-hole horizon.

67. E.W. Leaver, Phys. Rev. D 41, 2986 (1990)

68. H. Onozawa, T. Mishima, T. Okamura, H. Ishihara, Phys. Rev. D 53, 7033 (1996)

69. N. Andersson, H. Onozawa, Phys. Rev. D 54, 7470 (1996)

70. B.F. Schutz, C.M. Will, Astrophys. J. 291, L33 (1985)

71. S. Iyer, C.M. Will, Phys. Rev. D 35, 3621 (1987) 